\title{
Progranulin induces adipose insulin resistance and autophagic imbalance via TNFR1 in mice
}

\author{
Bo Zhou*, Huixia Li*, Jiali Liu, Lin Xu, Qinyue Guo, Hongzhi Sun and Shufang Wu \\ The First Affiliated Hospital of Medical School of Xi'an Jiaotong University, 277 West Yanta Road, Xi'an, \\ Shaanxi, China 710061 \\ *(B Zhou and $\mathrm{H}$ Li contributed equally to this work)
}

Correspondence should be addressed to $\mathrm{H}$ Sun or S Wu Email

shufangw@hotmail.com or sunhongzhi@mail.xjtu.edu.cn

\begin{abstract}
Progranulin (PGRN) has recently emerged as an important regulator for insulin resistance. However, the direct effect of PGRN in vivo and the underlying role of progranulin in adipose insulin resistance involving the autophagy mechanism is not fully understood. In this study, mice treated with PGRN for 21 days exhibited the impaired glucose tolerance and insulin sensitivity, remarkable adipose autophagy as well as attenuated insulin signaling via inhibition of mammalian target of rapamycin (mTOR) pathway. Furthermore, blockade of tumor necrosis factor receptor 1 (TNFR1) by TNFR1BP-Fc injection resulted in the restoration of impaired insulin sensitivity and insulin signaling induced by PGRN. Consistent with these findings in vivo, PGRN treatment induced defective insulin signaling, abnormal autophagic and mitochondrial activity in cultured adipocytes, while such effects were nullified by the blockade of TNFR1. In addition, PGRN-deficient adipocytes were more refractory to tunicamycin- or dexamethasone-induced insulin resistance, indicating the causative role of the TNFR1 pathway in the action of PGRN. Collectively, our findings support the notion that PGRN is a key regulator of insulin resistance and that PGRN may mediate its effects, at least in part, by inducing autophagy via the TNFR1-dependent mechanism.
\end{abstract}

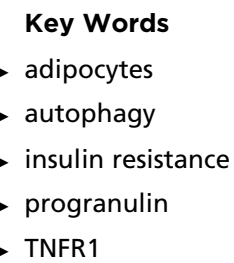

Journal of Molecular Endocrinology (2015) 55, 231-243

\section{Introduction}

Progranulin (PGRN), also known as proepithelin, granulin/ epithelin precursor or PC cell-derived growth factor, has recently emerged as an important regulator of glucose metabolism and insulin sensitivity. These studies showed that PGRN-deficient mice exhibited resistance to dietinduced obesity and insulin insensitivity through the modulation of inflammation, whereas adipocytes exposed to PGRN have increased susceptibility to be insulinresistant and those effects can be normalized with treatment of pioglitazone, an insulin-sensitizing agent (Matsubara \& Mita 2012). In particular, PGRN is more highly expressed in visceral adipose tissue of the insulinresistant patients with morbid obesity than in their age-, sex- and BMI-matched insulin-sensitive counterparts (Youn et al. 2009), suggesting that progranulin can be a key adipokine to mediate insulin sensitivity and energy metabolism.

Although the role of progranulin in adipose tissue in obesity is not fully understood, its link with autophagy in other physiological and pathological processes in other cell types has now been identified. Recent studies revealed an abnormal accumulation of lipofuscin granules in mice

Published by Bioscientifica Ltd 
brain regions with ablation of progranulin, accompanied by significantly increased p62 (Bjørkøy et al. 2005, Wils et al. 2012), suggesting impairment of the autophagylysosomal system in mice brains with ablation of progranulin. Furthermore, autophagy has been closely linked to inflammation in part by regulation of adipokine production. For example, mice with a conditional deletion of Atg7 in the intestinal epithelium showed an enhanced mRNA expression of IL1B (Crisan et al. 2011). Additional studies in vivo demonstrated that obesity-induced endoplasmic reticulum stress causes chronic inflammation in adipose tissue, as evidenced by up-regulation of TNFA, IL1B and IL6 (Kawasaki et al. 2012). These observations raised the possibility that PGRN might be a promising new link among obesity, autophagy and insulin resistance.

Though the progranulin membrane receptor has not yet been identified, it is important to clearly define the early stages of progranulin-mediated signaling from the plasma membrane. Answers to these questions are of critical importance for two reasons. First, if progranulin has an effect in WT mice, it would firmly establish the critical role of progranulin in the pathogenesis of metabolic disorders; second, it would address the therapeutic potential of this new player in the regulation of energy metabolism. In this study, we therefore aimed to evaluate the potential role of progranulin and its predominant receptor in adipose tissue in vivo. We also provided the evidences that autophagic activity and intracellular events required for progranulin-mediated effects in adipocytes. Our results support the hypothesis that progranulin is a novel adipokine that aggravates insulin resistance via the activated autophagy and impaired insulin signaling in a TNFR1-dependent manner, indicating the importance of this novel player in the regulation of glucose metabolism and a promising therapeutic target in the treatment of metabolic diseases.

\section{Material and methods}

\section{Preparation of recombinant mouse progranulin}

The pFLAG-CMV1 vector was purchased from Addgene (Cambridge, MA, USA). pCAGIPuro was constructed by replacing the zeocin-resistant gene of pPCAGIZ with the puromycin-resistant gene. pCAGIPuro-FLAG was constructed by subcloning the insert encoding the preprotrypsin signal peptide and FLAG epitope of pFLAG-CMV1 into pCAGIPuro. pCAGIPuro-FLAG was constructed by Sangon Biotech (Shanghai, China). pCAGIPuroFLAGmPGRN was constructed by sub-cloning the insert encoding the mPGRN without signal peptide (amino acid 18-589) into pCAGIPuro-FLAG. Then we have transfected the pCAGIPuro-FLAG-mPGRN construct into CHO-K1 cells by electroporation (BTX, Holliston, MA, USA). The culture supernatants were collected and subjected to anti-FLAG M1 agarose affinity gel column (Sigma, A4596). Then the recombinant PGRN samples were analyzed by SDS-PAGE and coomassie blue staining. The protein bands were visualized at $88 \mathrm{KD}$. Moreover, we used the mouse monoclonal anti-flag M1 antibody (Sigma, F3040) in immunoblot analyses, and the result showed that the full-length recombinant PGRN appeared as an 88 kDa FLAG-immunoreactive species. Finally, the purified PGRN were made endotoxin-free using the Detoxi-Gel endotoxin-removing column (Thermo Scientific, Rockford, IL, USA) as recommended by the manufacturer. As shown in Supplemental Fig. 1A, B and C, see section on supplementary data given at the end of this article, full-length progranulin can be found in $88 \mathrm{KD}$ and adipocytes exposed to full-length progranulin showed considerable attenuation in insulin signaling and increase in autophagic activity, whereas elastase-digested PGRN had little effect on insulin signaling and autophagy in adipocytes.

\section{Production of IgG1 Fc fused TNFR1 blocking peptide (TNFR1BP-FC)}

$4 \mu \mathrm{g}$ of $\mathrm{pIg} / 3 \mathrm{C}-\mathrm{TNFR} 1 \mathrm{BP}-\mathrm{Fc}$ plasmid or $\mathrm{pIg} / 3 \mathrm{C}$ plasmid were co-transfected along with a pcDNA3.1 vector that contained a Neo gene into CHO-K1 cells using Lipofectamine 2000 as instructed (Invitrogen). After $24 \mathrm{~h}$ of transfection, the cells were selected with $800 \mu \mathrm{g} / \mathrm{ml} \mathrm{G418}$ (GIBCO, Grand Island, NY, USA) for 8 days. Single clones were obtained by limited dilution and maintained in the presence of G418.

TNFR1BP-Fc or IgG1Fc (as a control) was purified from the culture supernatants by chromatography using protein A-Sepharose CL-4B beads (Amersham Biosciences, Uppsala, Sweden). The unpurified and purified proteins were analyzed on 10\% SDS-PAGE and stained with coomassie blue R250. The purified proteins were further passed through $0.45-\mu \mathrm{m}$ filters (Millipore, Boston, MA, USA) for sterilization as described (Liang et al. 2008).

\section{Animal care}

The experimental procedures performed in this study were in accordance with the guidelines of the Institutional Animal Ethics Committee for the Care and Use of Laboratory Animals. For the in vivo study, C57BL/6J male

Published by Bioscientifica Ltd. 
mice ( 8 weeks old) were fed with a normal chow diet and housed under standard conditions with a $12 \mathrm{~h}$ light: $12 \mathrm{~h}$ darkness cycle (darkness from 1930 to $0730 \mathrm{~h}$ ). Mice were distributed in four groups ( $n=10 /$ group): i) vehicle (normal saline solution); ii) progranulin (i.p. $1 \mu \mathrm{g} / \mathrm{g}$, once a day); iii) progranulin (i.p. $1 \mu \mathrm{g} / \mathrm{g}$, once a day) + IgG1Fc (injection via tail vein, $1 \mu \mathrm{g} / \mathrm{g}$, every three days); iv) progranulin (i.p. $1 \mu \mathrm{g} / \mathrm{g}$, once a day) + TNFR1BP-Fc (injection via tail vein, $1 \mu \mathrm{g} / \mathrm{g}$, every 3 days). The treatment lasted 21 days. At the end of the 21-day study period, half of the mice in each group were randomly selected and received an intraperitoneal injection of insulin at a dosage of $2 \mathrm{IU} / \mathrm{kg}$; 15 min after the injection, the animals were euthanized, and their omental adipose tissues and blood samples were obtained and stored at $-80^{\circ} \mathrm{C}$ for subsequent analysis. To measure food intake, mice were individually housed in metabolic cages; they were given an unlimited supply of food and allowed to feed ad libitum. Food consumption was measured daily and mean daily consumption was calculated at the end of study.

\section{Isolation and incubation of mouse adipocytes}

Adipocytes were isolated from omental adipose tissue by collagenase (Type 1, Worthington Biochemical, Lakewood, NJ, USA) digestion. Cells were treated and incubated in supplemented Krebs-Ringer solution as described (Stralfors \& Honnor 1989, Danielsson et al. 2005).

\section{Cell culture}

3T3-L1 cells were purchased from the American Type Culture Collection (ATCC, Manassas, VA, USA) and cultured in DMEM with $10 \%$ fetal bovine serum (FBS) (HyClone, Thermo Fisher Scientific, Inc. Logan, UT, USA). 3T3-L1 cells were induced to differentiate mature 3T3-L1 adipocytes with induction media by utilizing a standard protocol as described (Zhou et al. 2013a,b).

\section{Measurement of blood parameters}

Peripheral serum was subject to ELISA using standard kits (R\&D Systems, Inc., Minneapolis, MN, USA) for progranulin. Morning blood glucose and insulin levels were measured and glucose tolerance testing (GTT) and insulin tolerance testing (ITT) were performed by utilizing a standard protocol as described (Zhou et al. 2013a,b). Glucose tolerance testing (GTT) was performed after the mice were fasted overnight. A total of $2 \mathrm{~g} / \mathrm{kg}$ glucose was administrated through an i.p. injection, and blood glucose was measured at the indicated time points. Insulin tolerance testing (ITT) was performed after the animals had fasted for $4 \mathrm{~h}$. Then, $0.75 \mathrm{U} / \mathrm{kg}$ insulin was administered via i.p. injection, and blood glucose was measured at the indicated time points.

\section{Electron microscopy analysis}

Electron microscopy (EM) analysis was performed in omental adipose tissue samples by utilizing a standard protocol as described (Zhou et al. 2013a,b). Aorta samples were fixed in $4 \%$ paraformaldehyde/ $2 \%$ glutaraldehyde/ $0.1 \mathrm{M}$ sodium cacodylate $\mathrm{pH} 7.3$, post-fixed in $1 \%$ osmium tetraoxide and embedded in epoxy resin (Epon). Ultrathin sections $(80 \mathrm{~nm})$ were stained with aqueous uranyl acetate and lead citrate and examined with a JEOL 2000FX transmission electron microscope (JEOL). For quantification of autophagolysosome-like vacuoles, the numbers of autophagolysosomal-like vacuoles were counted in each field and normalized by the surface area. Sixteen electron micrographs per mouse were digitized and the area and number of clearly distinguishable mitochondria were analyzed using OsteoMeasure software (OsteoMetrics, Decatur, GA, USA).

\section{Glucose uptake}

After transfer of 3T3L1 cells to medium without glucose, mouse adipocytes were incubated with $10 \mathrm{nmol} / \mathrm{l}$ insulin for $15 \mathrm{~min}$, when glucose transport was determined as uptake of $50 \mu \mathrm{mol} / \mathrm{l}(10 \mu \mathrm{Ci} / \mathrm{ml}) 2$-deoxy-D-[1-3H] glucose, and then incubated $30 \mathrm{~min}$. Uptake was linear for at least $30 \mathrm{~min}$.

\section{Gene silencing and gene expression analysis}

Cells were transfected with a siRNA targeted for mouse PGRN (Santa Cruz, catalog number sc-39262), mouse TNFR1 (Santa Cruz, catalog number sc-36688) and mouse Atg7 (Santa Cruz, catalog number sc-41448 and Sigma, EMU094061) using Lipofectamine 2000 (Invitrogen). Gene silencing and real-time PCR was performed by utilizing a standard protocol as described (Zhou et al. 2013a,b). The real-time PCR primers included:

Atg7 For: GTT CGC CCC CTT TAA TAG TGC, Rev: TGA ACT CCA ACG TCA AGCGG; p62 For: AGG ATG GGG ACT TGG TTGC, Rev: TCA CAG ATC ACA TTG GGG TGC; UCP1 For: AAG CGT ACC AAG CTG TGCGA, Rev: AGAAAAGAAGCCACAAACCCTT; Cebpa For: GAA TCT CCT AGT CCT GGCTC, Rev: GAT GAGAACAGCAACGAGTAC;

Published by Bioscientifica Ltd. 
FABP4 For: ATG ATC ATC AGC GTA AATGG, Rev: GCC TTT CAT AAC ACA TTCCA; Il6 For: ACA ACCACGGCCTTCCCTACTT, Rev: CACGATTTCCCAGAGAACATGTG; Pgc1a For:AGC CAA ACC AAC AAC TTT ATC TCTTC, Rev: TTA AGG TTC GCT CAA TAG TCT TGTTC.

\section{Western blot}

Tissues and cells under various treatments were lysed in lysis buffer containing $25 \mathrm{mM}$ Tris- $\mathrm{HCl}$ (pH 6.8), 2\% SDS, 6\% glycerol, 1\% 2-mercaptoethanol, $2 \mathrm{mM}$ phenylmethylsulfonyl fluoride, $0.2 \%$ bromophenol blue and a protease inhibitor cocktail for $20 \mathrm{~min}$. Western blotting was performed by utilizing a standard protocol as described (Wu et al. 2009).

\section{Antibodies}

The following antibodies were used: anti-Atg7, anti-p62, anti-LC3, anti-p-PERK, anti-PERK, anti-p-eIF2A and antieIF2A (Cell Signaling Technology Inc. Danvers, MA); anti-IRS-1, anti-pY20, anti-p-Akt, anti-Akt, anti-p-mTOR, anti-mTOR, anti-PGRN, anti-GAPDH (Santa Cruz Biotechnology, Inc.).

\section{Statistical analysis}

Statistical analyses were performed using SPSS 17.0 Software. Statistical analysis between the two groups was performed using unpaired, two-tailed Student $t$-test or ANOVA. Differences were considered significant when the $P$ value was $<0.05$.

\section{Results}

\section{Effects of recombinant mouse progranulin on glucose metabolism, insulin sensitivity and mitochondrial activity via the TNFR1-dependent mechanism in vivo}

To evaluate the role of progranulin in insulin resistance, recombinant mouse progranulin was administrated intraperitoneally to mice under standard diet condition. We found that serum progranulin levels increased about twofold and the protein level of progranulin was also elevated in adipose tissue after treatment with progranulin for 21 days (Fig. 1A and Supplemental Fig. 1D, see section on supplementary data given at the end of this article). Although body weight, fat pads weight, food intake, fasting insulin level and blood glucose level were not significantly different (Fig. 1B, C, D and 1E, Supplemental
Fig. 1E), mice treated with progranulin exhibited decreased glucose tolerance and insulin sensitivity, as assessed by GTT and ITT (Fig. 1I and J), suggesting that progranulin may directly cause insulin resistance in vivo. However, injection with progranulin and TNFR1BP-Fc in mice was associated with improved glucose tolerance and insulin sensitivity compared with mice injected with progranulin and IgG1Fc (Fig. 1I and J, Supplemental Fig. 1F), and no significant difference was observed with respect to body weight, food intake, fasting insulin level, blood glucose level or serum TNFA level between the two groups (Fig. 1B, C, D and E, Supplemental Fig. 1G).

Next, we further performed molecular and histomorphometric analysis in adipose tissue. Expression of $P_{g c 1 a}$ and Ucp1, molecular markers of mitochondrial function, was significantly reduced in adipose tissue of mice receiving progranulin compared to that of vehicleinjected mice (Fig. 1F and Supplemental Fig. 1H, see section on supplementary data given at the end of this article). The alteration of Pgc1a and Ucp1 expression indicated that progranulin treatment inhibited mitochondrial activity in the adipose tissue of mice. Consistent with these findings, morphology analysis of adipose tissue by EM showed decreased number and area of mitochondria in mice injected with progranulin compared with those of vehicle-injected mice ( $\mathrm{G}$ and $1 \mathrm{H}$ ). Meanwhile, the reduction in the expression level of $P g c 1 a$ and $U c p 1$ as well as the reduction in the number and the area of mitochondria in adipose tissue of mice injected with progranulin was significantly neutralized by TNFR1BP-Fc. Moreover, we examined the expressions of adipogenic genes (Cebpa), adipose-specific genes (Fabp4), and inflammatory adipokines (Il6) in adipose tissue, which are involved in the development of insulin resistance. Progranulin injection increased Il6 expression, reduced Cebpa and Fabp4 expression, with these effects of progranulin being reversed by TNFR1BP-Fc (Supplemental Fig. 1I, J and K, see section on supplementary data given at the end of this article). These findings indicated that TNFR1BP-Fc partially blocks the effect of progranulin in vivo.

\section{Regulation of progranulin in autophagy and insulin signaling pathway via the TNFR1-dependent mechanism in adipose tissue of mice}

To understand the mechanisms for the effect of progranulin on insulin sensitivity and mitochondrial activity, we investigated whether progranulin could activate autophagy and impair insulin receptor signaling in mice. We first analyzed mRNA and protein expression of autophagy

Published by Bioscientifica Ltd 

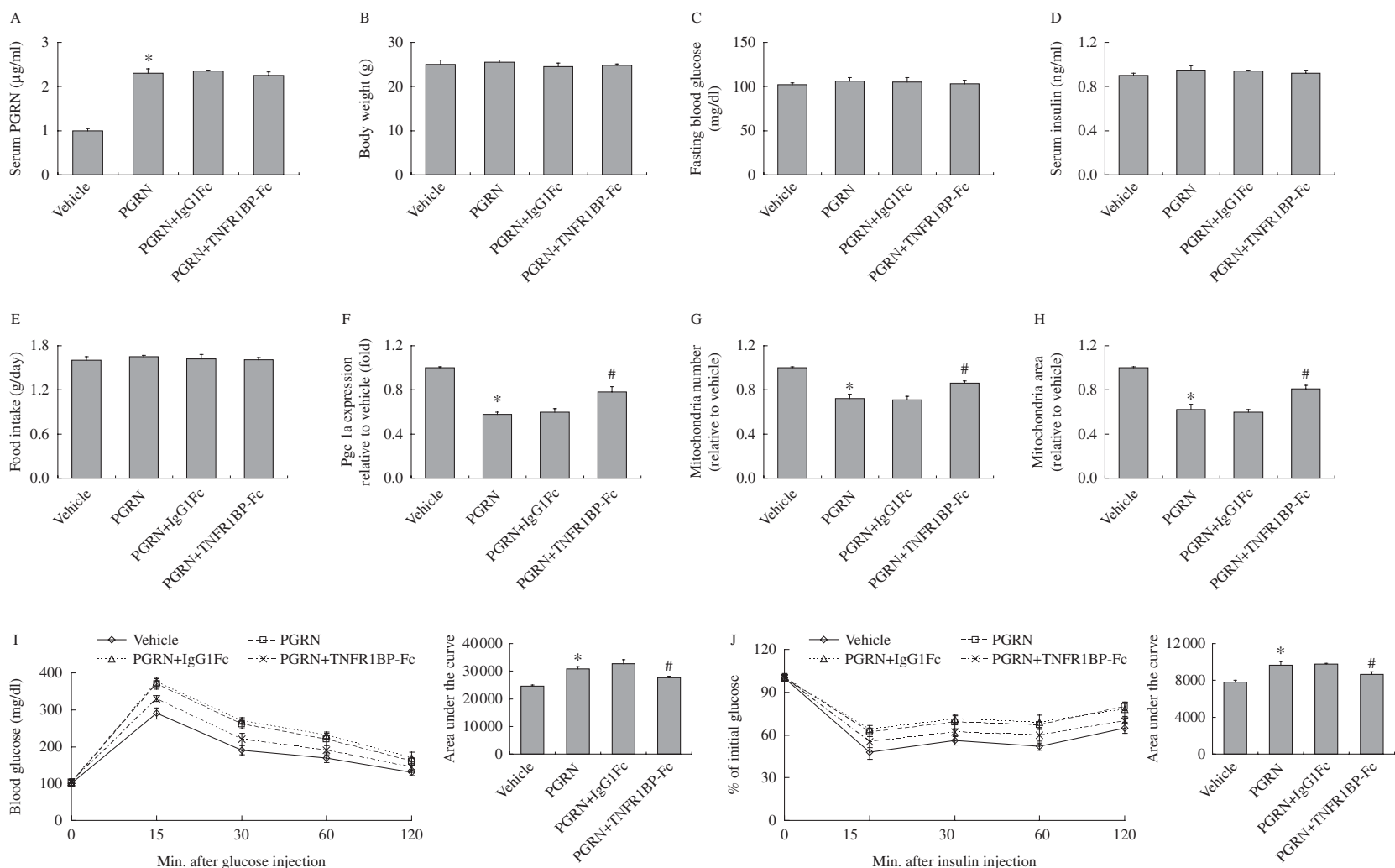

Figure 1

Effects of recombinant mouse progranulin (PGRN) on glucose metabolism, insulin sensitivity and mitochondrial activity via the TNFR1-dependent mechanism in vivo. All analyses compared age- and sex-matched mice fed a normal diet. Mice were divided into four groups: i) vehicle; ii) progranulin; iii) progranulin + IgG1Fc; iv) progranulin + TNFR1BP-Fc. (A) Serum progranulin. (B) Body weight. (C) Blood glucose. (D) Serum insulin. (E) Food

indicators by real-time PCR and western blotting in omental adipose tissue. We found that progranulin injection resulted in increased expression of autophagy indicators in omental adipose tissue of mice, as evidenced by upregulation of $A \operatorname{tg} 7 \mathrm{mRNA}$ and protein level, and light chain 3 (LC3)-II protein levels. In contrast, mRNA and protein expression of p62 was reduced (Fig. 2A, B and C). We also observed the decreased activity of mammalian target of rapamycin (mTOR), a key component that regulates autophagy in response to cellular physiological conditions, demonstrating upregulation of autophagy (Fig. 2C). However, TNFR1BP-Fc injection inhibited mRNA and protein expression of autophagy indicators in adipose tissue of the mice injected with progranulin (Fig. 2A, B and C). Simultaneously, electron microscopic examination of adipose tissue showed a significant increase in autophagosome/autolysosome formation in mice treated with progranulin as compared with vehicleinjected mice and a reduction in mice injected with

intake. (F) Relative expression of Pgc1alpha in adipose tissue (real-time PCR). (G) Mitochondria number in adipose tissue. (H) Mitochondria area in adipose tissue. (I) Glucose tolerance testing (GTT). (J) Insulin tolerance testing (ITT). Data are expressed as means \pm SD in each bar graph from ten mice per group. ${ }^{*} P<0.05$ (vehicle vs PGRN). ${ }^{*} P<0.05$ (PGRN + lgG1Fc vs PGRN + TNFR1BP-FC).

progranulin and TNFR1BP-Fc compared to the mice injected with progranulin and IgG1Fc (Fig. 2D and Supplemental Fig. 2A, see section on supplementary data given at the end of this article), supporting the biochemical alterations in key autophagy molecules.

Next, we investigated the expression of indicators of insulin receptor signaling by western blotting and immunoprecipitation. We found that insulin receptor signaling was markedly inhibited in omental adipose tissue of mice injected with progranulin, as shown by the reduction of IRS-1 tyrosine phosphorylation and, more distally, Akt Ser-473 phosphorylation (Fig. 2E). Nevertheless, insulin receptor signaling was restored in adipose tissue of mice receiving TNFR1BP-Fc (Fig. 2E).

Considering the possibility that the increased levels of autophagy markers such as LC3-II seen in omental adipose tissue of mice injected with progranulin may reflect a lower turnover rather than increased autophagosome production, we treated omental adipose tissue explants

Published by Bioscientifica Ltd 

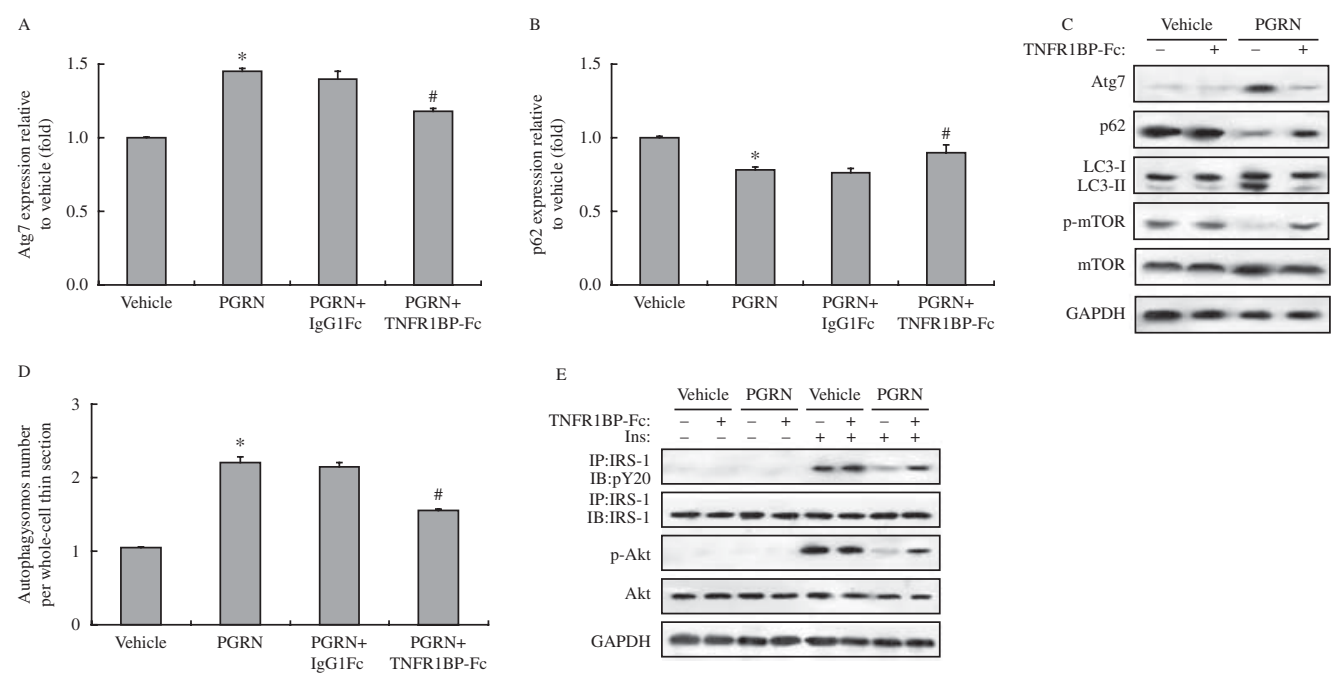

\section{Figure 2}

Regulation of progranulin (PGRN) in autophagy and insulin signaling pathway via the TNFR1-dependent mechanism in vivo. All analyses compared age- and sex-matched mice fed a normal diet. Mice were divided into four groups: i) vehicle; ii) progranulin; iii) progranulin + lgG1Fc; iv) progranulin + TNFR1BP-Fc. The relative quantity of proteins was analyzed using Quantity One Software. (A) Relative expression of Atg7 in adipose tissue (real-time PCR). (B) Relative expression of $p 62$ in adipose

with chloroquine (a lysosomal protease inhibitor) or bafilomycin (an autophagy inhibitor), and monitored the formation of autophagosome. Remarkably, the expression of p62 and LC3-II was increased in omental adipose tissue explants of predisposed with PGRN and chloroquine/bafilomycin compared to controls with chloroquine/bafilomycin (A and B). In addition, to further confirm the association between autophagy and insulin resistance in omental adipose tissue explants, we predisposed omental adipose tissue explants with pioglitazone, a PPARgamma agonist that improves insulin sensitivity. As expected, we also observed that the increase of autophagy indicators and the suppression of insulin signaling by progranulin were nullified by the addition of pioglitazone in omental adipose tissue explants (Fig. 3C and D). These findings indicated that injections of progranulin suppress insulin sensitivity in the adipose tissue possibly by the activation of autophagy in vivo.

\section{Effects of progranulin on autophagy and insulin sensitivity in 3T3-L1 adipocytes}

To identify the role of progranulin in insulin resistance in vitro, we first elucidated the effect of progranulin on autophagy in mature adipocytes. We treated mature 3T3-L1 adipocytes with $100 \mathrm{ng} / \mathrm{ml}$ progranulin for $20 \mathrm{~h}$ tissue (real-time PCR). (C) Protein expression of Atg7, p62 and LC3, and phosphorylation of mTOR in adipose tissue. (D) Quantification of autophagolysosome-like vacuoles per field in the EM images in adipose tissue. (E) Phosphorylation of IRS1 and $A k t$ in adipose tissue. Data are expressed as means \pm s.D. in each bar graph from ten mice per group and a representative blot is shown. ${ }^{*} P<0.05$ (vehicle vs PGRN). ${ }^{\#} P<0.05$ (PGRN + IgG1FC vs PGRN + TNFR1BP-FC).

and measured the protein expression of indicators of autophagy and insulin receptor signaling by western blot. Progranulin treatment, with or without insulin, not only significantly elevated protein expression of Atg7 and LC3-II, but also reduced mTOR phosphorylation and p62 expression (Fig. 4A), suggesting that protein levels of autophagy-related genes is mediated by progranulin in an insulin-independent manner. Since progranulin has been shown to be involved in the PI3K/Akt signaling pathway, we postulated that progranulin might indirectly affect insulin signaling via upregulation of autophagy indicators in mature adipocytes. As expected, insulin-stimulated phosphorylations of both IRS1 and Akt were decreased in mature adipocytes treated with progranulin (Fig. 4B). Furthermore, progranulin treatment significantly suppressed insulin-stimulated glucose uptake (Fig. 4C), and decreased the number and the area of mitochondria in 3T3-L1 adipocytes (Fig. 4D and E). Additionally, we also analyzed whether insulin resistance was due to increased autophagy directly. Atg7 siRNA was validated by measurement of reduced Atg7 protein expression by western blotting in Atg7 siRNA (Santa Cruz, catalog number sc-41448 and Sigma, EMU094061) transfected adipocytes (Fig. 4F and Supplemental Fig. 2B, C and D, see section on supplementary data given at the end of this article). As shown in Fig. 4G and H, and Supplemental Fig. 2C, D

Published by Bioscientifica Ltd. 


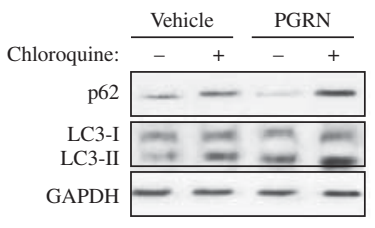

B

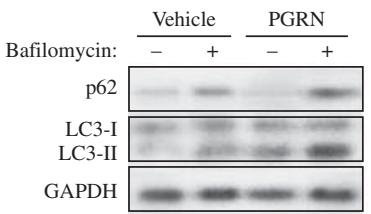

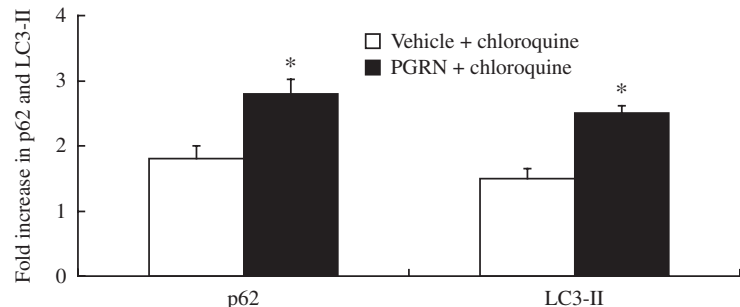
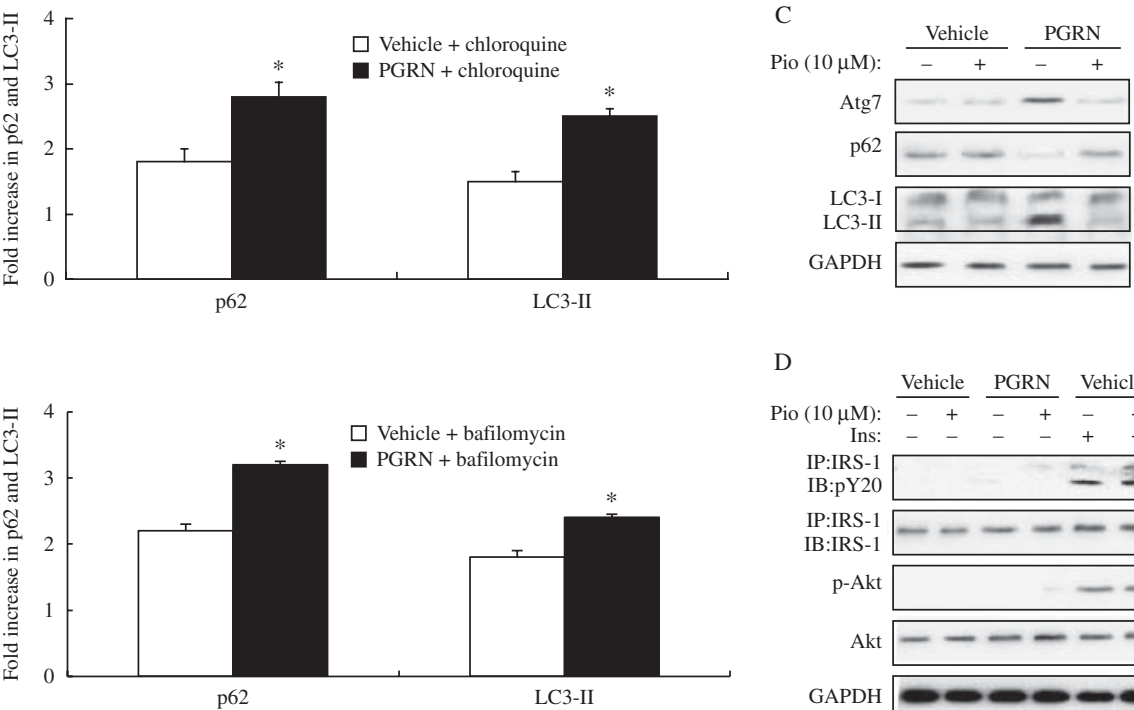

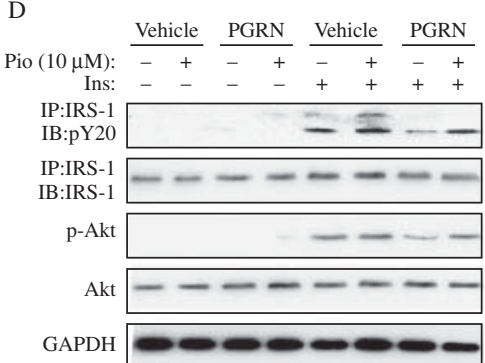

Figure 3

Regulation of progranulin in autophagy and insulin signaling pathway in omental adipose tissue explants. Analysis of autophagic flux using chloroquine or bafilomycin was performed in omental adipose tissue explants. For insulin signaling, cells were stimulated with $10 \mathrm{nM}$ of insulin for $10 \mathrm{~min}$. (A) Protein expression of $p 62$ and LC3 in omental adipose tissue explants treated with or without chloroquine. (B) Protein expression of $p 62$ and LC3 in omental adipose tissue explants treated with or without bafilomycin. (C) Protein expression of Atg7, p62 and LC3 in omental

and $\mathrm{E}$, see section on supplementary data given at the end of this article, treatment of progranulin reduced phosphorylation level of Akt and glucose uptake in adipocytes, while phosphorylation level of $A k t$ and glucose uptake had a significant increase in adipocytes co-treated with progranulin and Atg7 siRNA. Therefore, blockade of Atg7 partially reversed impaired insulin signaling and suppressed insulin-stimulated glucose uptake in adipocytes treated with progranulin.

\section{Ablation of progranulin prevents tunicamycin- or dexamethasone-induced autophagy and impaired insulin signaling in 3T3-L1 adipocytes}

To further confirm the action of progranulin on autophagy and insulin signaling, we first determined whether ablation of progranulin reverses tunicamycin-induced autophagy and impaired insulin signaling in mature 3T3-L1 adipocytes. Progranulin siRNA was validated by measurement of reduced progranulin protein expression by western blot in progranulin siRNA-transfected 3T3-L1 adipocytes (Fig. 5A, B, C and D). In the presence of tunicamycin, the expression of progranulin was elevated while effectively knocked down by concomitant transfection adipose tissue explants treated with or without $10 \mu \mathrm{M}$ pioglitazone (an insulin sensitizer). (D) Phosphorylation of IRS1 and Akt in omental adipose tissue explants treated with or without pioglitazone.

A representative blot from three independent experiments is shown and the data expressed as means \pm s.D. in each bar graph represent the average of three independent experiments. ${ }^{*} P<0.05$ (vehicle/chloroquine or bafilomycin vs PGRN/chloroquine or bafilomycin).

of progranulin siRNA in 3T3-L1 adipocytes (Fig. 5A). As expected, tunicamycin (an agent commonly used to induce endoplasmic reticulum stress, leading to insulin resistance) not only produced a significant increase in autophagy, as evidenced by up-regulation of Atg7 and LC3-II protein expression and downregulation of p62 expression (Fig. 5A), but also decreased insulin-stimulated tyrosine phosphorylation of IRS1 and Akt Ser-473 (Fig. 5B). However, blockade of progranulin by transfection with progranulin siRNA in adipocytes reversed the effect of tunicamycin (Fig. 5A and B). In addition, we found that ablation of progranulin also prevented dexamethasone(an agent commonly used to induce insulin resistance) induced autophagy and impaired insulin signaling in 3T3-L1 adipocytes (Fig. 5C and D). These observations further identified the effect of progranulin on insulin sensitivity in 3T3-L1 adipocytes.

\section{TNFR1BP-Fc or TNFR1 siRNA blocks the effect of TNFR1-mediated progranulin in 3T3-L1 adipocytes}

Based on our findings that TNFR1 is involved in the regulation of progranulin function in vivo, we postulated that TNFR1 could be a specific receptor for progranulin.

Published by Bioscientifica Ltd. 
A

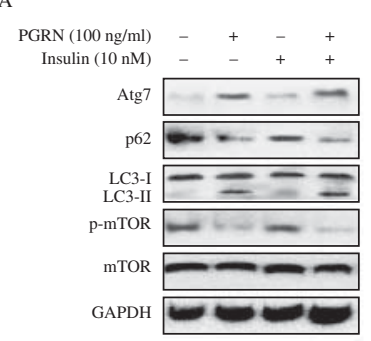

B

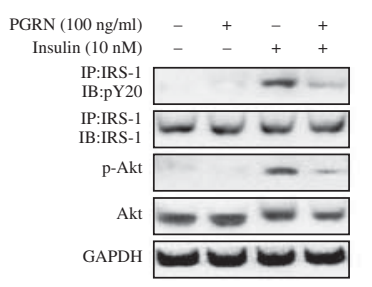

C

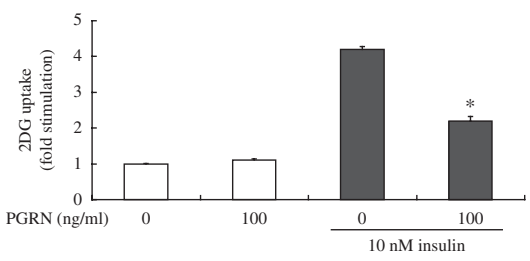

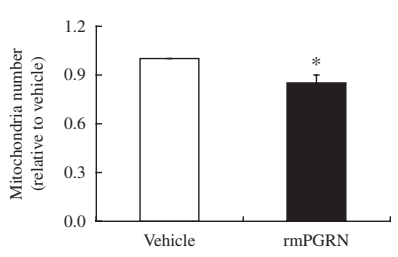

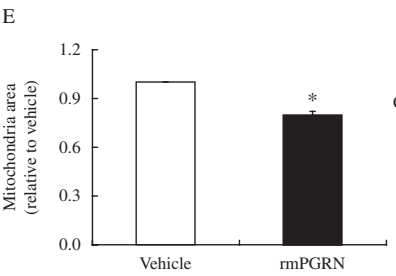

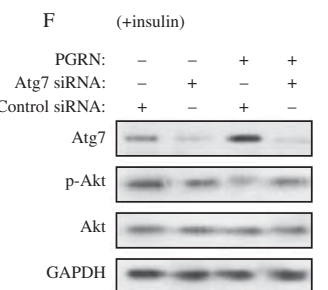

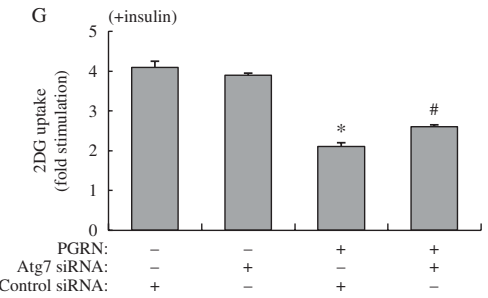

Figure 4

Effects of progranulin on autophagy and insulin sensitivity in 3T3-L1 adipocytes. 3T3-L1 adipocytes were cultured in the presence or absence of progranulin (PGRN) $100 \mathrm{ng} / \mathrm{ml}$ for $20 \mathrm{~h}$ with or without $A \operatorname{tg} 7$ siRNA (Santa Cruz, catalog number sc-41448). (A) Protein expression of Atg7, p62 and LC3, and phosphorylation of mTOR. (B) Phosphorylation of IRS1 and Akt. (C) Effects of progranulin on glucose uptake. (D) Mitochondria number. (E) Mitochondria area. (F) Protein expression of Atg 7 and

To confirm the hypothesis, we suppressed TNFR1 expression via TNFR1BP-Fc and TNFR1 siRNA in mature 3T3-L1 adipocytes in the presence of progranulin. TNFR1 siRNA-transfected 3T3-L1 adipocytes exhibited reduced TNFR1 protein expression compared with control siRNAtransfected 3T3-L1 adipocytes (Fig. 7A). Progranulin treatment reduced mTOR phosphorylation and insulinstimulated glucose uptake, increased protein expression of
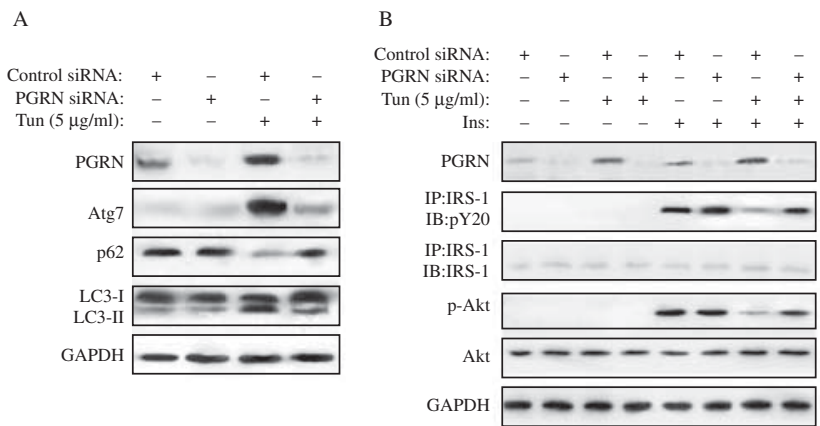

phosphorylation of $A k t$ in adipocytes treated with Atg7 siRNA (Santa Cruz, catalog number sc-41448). (G) Effects of Atg7 siRNA (Santa Cruz, catalog number sc-41448) on glucose uptake. A representative blot from three independent experiments is shown and the data expressed as means \pm s.D. in each bar graph represent the average of three independent experiments. ${ }^{*} P<0.05$ (vehicle vs PGRN or control siRNA vs PGRN). ${ }^{\#} P<0.05$ (PGRN + control siRNA vs PGRN + Atg7 siRNA).

autophagy indicators and autophagysomos number, impaired insulin signaling and induced insulin resistance, but blockade of TNFR1 by the addition of TNFR1BP-Fc in the culture medium or transfection with TNFR1 siRNA nullified the effect of progranulin in 3T3-L1 adipocytes (Fig. 6A, B, C, D, E and F, Fig. 7A, B, C, D, E and F; Supplemental Fig. 2F and G, see section on supplementary data given at the end of this article). Furthermore, we also
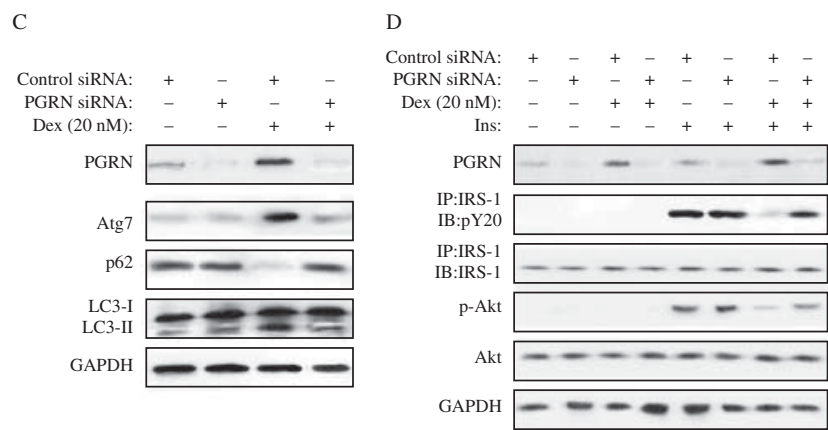

Figure 5

Ablation of progranulin prevents tunicamycin- or dexamethasone- induced autophagy and impaired insulin signaling in 3T3-L1 adipocytes. 3T3-L1 adipocytes were cultured in the presence or absence of $100 \mathrm{nM}$ progranulin (PGRN) siRNA. (A) Protein expression of progranulin, Atg7, p62 and LC3 in adipocytes treated with tunicamycin. (B) Protein expression of progranulin and phosphorylation of IRS1 and Akt in adipocytes treated with

tunicamycin. (C) Protein expression of progranulin, Atg7, p62 and LC3 in adipocytes treated with dexamethasone. (D) Protein expression of progranulin and phosphorylation of IRS1 and $A k t$ in adipocytes treated with dexamethasone. A representative blot from three independent experiments is shown.

Published by Bioscientifica Ltd. 


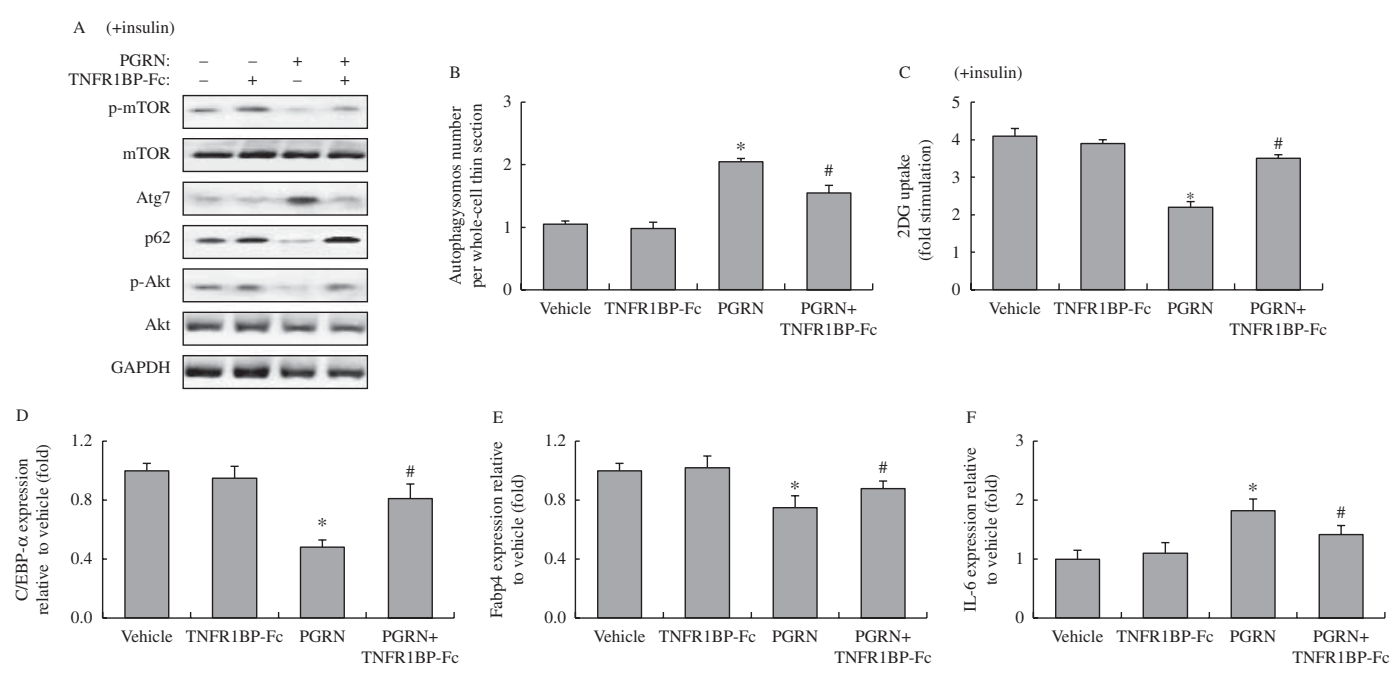

\section{Figure 6}

TNFR1BP-Fc blocks the effect of TNFR1-mediated progranulin in 3T3-L1 adipocytes. (A) Protein expression of Atg7 and $p 62$ and the phosphorylation of mTOR and Akt in 3T3-L1 adipocytes in the presence or absence of progranulin (PGRN) with or without $100 \mathrm{ng} / \mathrm{ml}$ TNFR1BP-Fc. (B) Autophagysomos number. (C) Glucose uptake. (D) Relative expression of Cebpa

tested the effect of a TNFA antagonist (also designated WP9QY), which is an exocyclic peptidomimetic that acts as a TNFA antagonist by mimicking the most critical recognition loop on TNFR1. As shown in Supplemental Fig. $2 \mathrm{H}$, I and J, see section on supplementary data given at the end of this article, the TNFA antagonist reversed the effect of progranulin in 3T3-L1 adipocytes and no significant difference of TNFA level was observed in the medium of 3T3-L1 adipocytes with or without progranulin. Meanwhile, the interaction between PGRN and TNFR1 in adipocytes was also demonstrated by co-immunoprecipitation (Co-IP) (Supplemental Fig. 2K), indicating that progranulin activated autophagy and inhibited insulin sensitivity, at least in part, by binding to TNFR1 in 3T3-L1 adipocytes.

\section{Discussion}

Recent evidence indicated that progranulin mediates high-fat-diet-induced insulin resistance and obesity through inducing the expression of Il6, and ablation of progranulin protected against high-fat-diet-induced obesity and insulin resistance in vivo (Matsubara \& Mita 2012). Consistent with these findings, we also found that mice injected with progranulin developed glucose intolerance and insulin insensitivity. Of note, the respiratory quotient in mice with ablation of progranulin fed a high-fat diet (real-time PCR). (E) Relative expression of Fabp4 (real-time PCR). (F) Relative expression of $/ 16$ (real-time PCR). A representative blot from three independent experiments is shown and the data expressed as means \pm s.D. in each bar graph represent the average of three independent experiments. ${ }^{*} P<0.05$ (vehicle vs PGRN). ${ }^{\#} P<0.05$ (PGRN vs PGRN + TNFR1BP-Fc).

was significantly lower at dark phase (Matsubara \& Mita 2012), suggesting that ablation of progranulin suppressed high-fat-diet-induced obesity by consuming lipids more preferentially than carbohydrate. In line with these results in vivo, we also found that ablation of progranulin reverses tunicamycin-induced autophagy and impaired insulin signaling in mature adipocytes. Accordingly, progranulin may partly participate in chronic inflammation associated with insulin resistance and obesity, but the definite effect of progranulin in humans remains to be determined.

A recent study implicates the causative role of progranulin in the pathogenesis of the adipose insulin resistance of obese mice (He et al. 2002). However, the mechanisms of this defect could be diverse. Since obesity is characterized with enhanced intracellular lipid accumulation (Ost et al. 2010), the role of progranulin with sustained lipogenesis might be much more complicated than expected. It is plausible that chronic lipid overloading, which might impair insulin signaling and insulin-stimulated glucose uptake, might be one of the triggers in metabolic disturbance. Thus, systematic investigation of the metabolic consequences of progranulin administration is warranted in animals with the absence of other chronic changes that accompany the obese state. Our study demonstrated that mice treated with progranulin under standard diet conditions exhibited abnormal ITT and impaired insulin signaling with autophagy

Published by Bioscientifica Ltd 


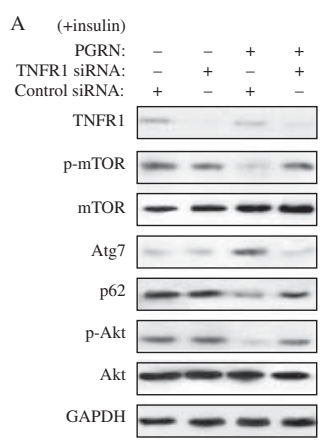

D

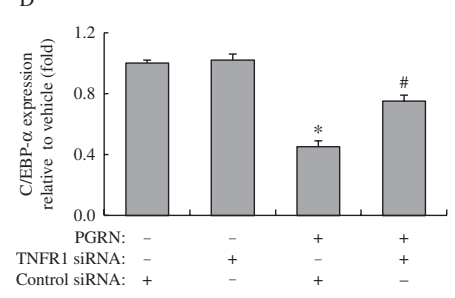

B

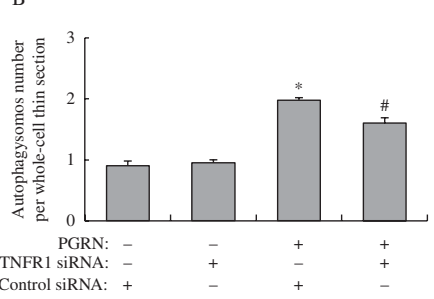

TNFR1 siRNA:
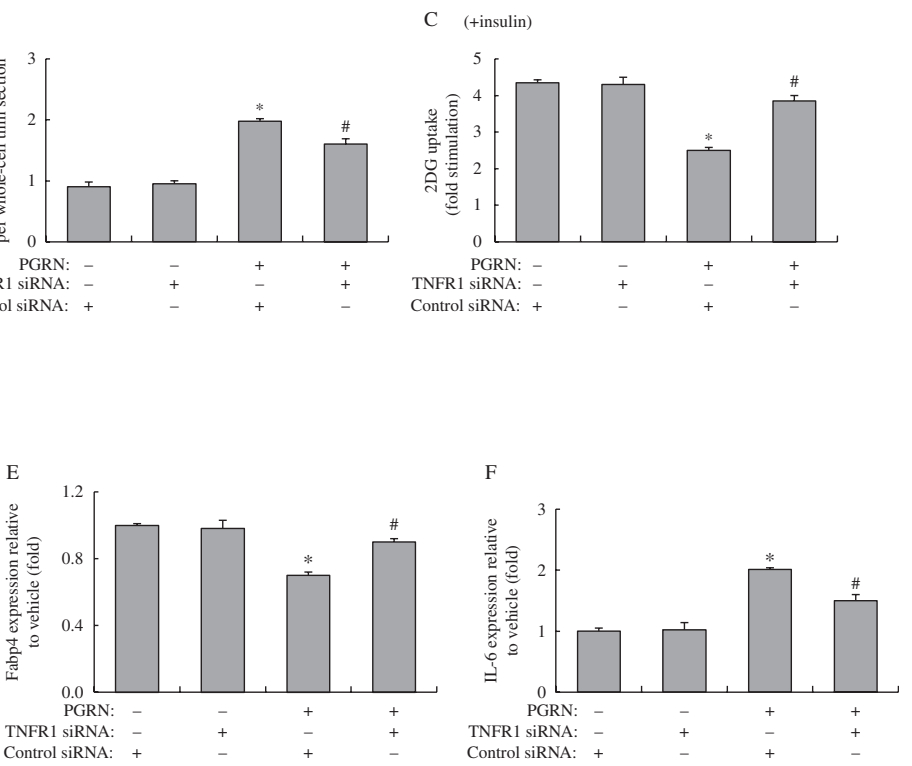

\section{Figure 7}

TNFR1 siRNA blocks the effect of TNFR1-mediated progranulin in 3T3-L1 adipocytes. (A) Protein expression of TNFR1, Atg7 and p62, and the phosphorylation of mTOR and Akt in 3T3-L1 adipocytes in the presence or absence of progranulin (PGRN) with or without $100 \mathrm{nM}$ TNFR1 siRNA. (B) Autophagysomos number. (C) Glucose uptake. (D) Relative expression of Cebpa (real-time PCR). (E) Relative expression of Fabp4 (real-time PCR).
(F) Relative expression of $1 / 6$ (real-time PCR). A representative blot from three independent experiments is shown and the data expressed as means \pm S.D. in each bar graph represent the average of three independent experiments. ${ }^{*} P<0.05$ (control siRNA vs PGRN). ${ }^{\#} P<0.05$ (PGRN + control siRNA vs PGRN + TNFR1 siRNA).

2008). Consistent with these findings, we also showed that blockade of TNFR1 partially reversed progranulin-induced insulin resistance. It has been well demonstrated that blockade of TNFR1 signaling protected Wistar rats from high-fat-diet-induced obesity and adiposity, and TNFA neutralizing antibodies have been used to block TNFA bioactivity clinically as a therapeutic approach for obesity and insulin resistance (Liang et al. 2008). As indicated earlier, the expression of progranulin in blood and adipose tissues was markedly increased in obese mouse, and even ablation of progranulin prevented mice from high-fatdiet-induced obesity (Matsubara \& Mita 2012). In our analysis, PGRN caused impaired insulin signaling mainly through TNFR1 rather than TNFR2 in vitro. Several other groups also independently reproduced the binding of PGRN to TNFR1 and TNFR2 and the inhibitory effect of this binding on TNFA-induced effects (Jian et al. 2013, Hu et al. 2014, Liu et al. 2014). Our findings support the notion that PGRN is a key regulator of insulin resistance and that PGRN may mediate its effects, at least in part, by binding to TNF receptors.

It has been reported that mitochondrial dysfunction has been implicated in the pathogenesis of type 2 diabetes and insulin resistance. Increased intracellular fat 
accumulation in the liver and skeletal muscle leads to impaired activity of mitochondrial oxidative phosphorylation and insulin resistance in high-fat-diet-induced obese mice (Petersen et al. 2003). Microarray analysis studies have recently shown that the expression of genes involved in mitochondrial oxidative metabolism is reduced in humans with insulin resistance and diabetes (Mootha et al. 2003, Patti et al. 2003). For example, Pgc1a-responsive genes involved in oxidative phosphorylation are coordinately downregulated in human diabetes. In our study, expression of $P g c 1 a$ was significantly reduced in adipose tissue of mice receiving progranulin compared to that of vehicle-injected mice. Simultaneously, histomorphometric quantification also showed a decrease in the number and the area of mitochondria in the mice injected with progranulin. Based on these results, we postulated that mitochondrial dysfunction and insulin resistance are interrelated. Notably, it has been shown that mitochondrial flavoprotein apoptosis-inducing factor-knockout mice with impaired mitochondria activity are resistant to high-fat-diet-induced obesity and diabetes (Pospisilik et al. 2007), indicating that the relationship between mitochondrial function and insulin sensitivity is not straightforward.

TNFR1 and TNFR2 do not share homology in the cytoplasmic domains but exhibit a low degree of similarity in the ligand-binding region located in the extracellular domains, which suggests that they are capable of inducing distinct cellular responses (Liang et al. 2008). Some studies imply PGRN elicits its action more through TNFR2 than TNFR1, because disturbed the interaction of PGRN with TNFR2, and in turn abolished PGRN-mediated activation of Erk1/2 and Akt signaling and protection against apoptosis in response to ER-stress (Li et al. 2014). Autophagy induction is an indirect message that protein synthesis is in suspended state, and it is possible that it could benefit in restoration of activated ER stress due to unfolded protein response (Komatsu et al. 2005, Mortensen et al. 2010), thus we extrapolated enhanced autophagy may be a protective and de-compensatory response to increased ER stress or a process underlying increased cell death. In other physiological and pathological processes, it is clear that protein expression of autophagy genes is increased in adipose tissue of humans and mice in obesity (Zhou et al. 2009, Kovsan et al. 2011), but impaired autophagy with decreased expression of autophagy genes has been shown in liver of obese mice and tied to insulin resistance (Yang et al. 2010), suggesting that the expression of autophagy genes is different in adipose tissue and the liver of obese mice. Additionally, recent results showed that PGRN may induce cholangiocyte proliferation by inhibiting autophagy via the suppression of Sirt1 expression (DeMorrow \& Francis 2015). It is possible that enhanced autophagy represents an integral part of the ER stress and contributes to be a protective response in adipose tissue of PGRN-injected mice. In parallel, our recent study indicated a complimentary effect of both TNFR1 and TNFR2 in mediating PGRN function in cultured adipocytes. These discrepancies might be possibly due the fact that there is a different distribution between TNFR1 and TNFR2 in different cell types, and the function of PGRN might be diverse in different tissues, which should warrant further investigations.

In conclusion, our present study revealed that administration of PGRN attenuated insulin signaling and triggered autophagy in vivo and in vitro studies, with such effects being drastically blocked by TNFR1BP-Fc, suggesting a causative role of TNFR1 in PGRN-induced impaired insulin sensitivity and implicating that decreasing PGRN levels by influencing its turnover or production is consequently a promising therapeutic approach applied to metabolic disorders.

\section{Supplementary data}

This is linked to the online version of the paper at http://dx.doi.org/10.1530/ JME-15-0075.

\section{Declaration of interest}

The authors declare that there is no conflict of interest that could be perceived as prejudicing the impartiality of the research reported.

\section{Funding}

This work was supported by the programs from the National Natural Science Foundation of China (General Program no. 81370899, no. 81170741, no. 81472038 and no. 81500016), National Excellent Young Scientist Program (no. 81222026) and the New Century Excellent Talents in University from the Ministry of Education, China (NCET-08-0435).

\section{Author contribution statement}

B Z wrote the manuscript and researched data. H L, J L, L X and Q G researched data and contributed to the discussion. S W and $\mathrm{H} S$ contributed to the experimental designs and reviewed and edited the manuscript. $\mathrm{HS}$ is the guarantor of this work and, as such, had full access to all the data in the study and takes responsibility for the integrity of the data and the accuracy of the data analysis.

\section{Acknowledgements}

We appreciate the technical support and materials from the electron microscope center of Xi'an Jiaotong University.

Published by Bioscientifica Ltd. 


\section{References}

Bjørkøy G, Lamark T, Brech A, Outzen H, Perander M, Overvatn A, Stenmark H \& Johansen T 2005 P62/SQSTM1 forms protein aggregates degraded by autophagy and has a protective effect on huntingtininduced cell death. Journal of Cell Biology 171 603-614.

Bluml S, Binder NB, Niederreiter B, Polzer K, Hayer S, Tauber S, Schett G, Scheinecker C, Kollias G, Selzer E et al. 2010 Antiinflammatory effects of tumor necrosis factor on hematopoietic cells in a murine model of erosive arthritis. Arthritis and Rheumatism 62 1608-1619. (doi:10.1002/ art.27399)

Chen X, Chang J, Deng Q, Xu J, Nguyen TA, Martens LH, Cenik B, Taylor G, Hudson KF, Chung J et al. 2013 Progranulin does not bind tumor necrosis factor (TNF) receptors and is not a direct regulator of TNFdependent signaling or bioactivity in immune or neuronal cells. Journal of Neuroscience 33 9202-9213. (doi:10.1523/JNEUROSCI. 5336-12.2013)

Crisan TO, Plantinga TS, van de Veerdonk FL, Farcas MF, Stoffels M, Kullberg BJ, van der Meer JW, Joosten LA \& Netea MG 2011 Inflammasome independent modulation of cytokine response by autophagy in human cells. PLOS ONE 6 e18666. (doi:10.1371/journal. pone.0018666)

Danielsson A, Ost A, Lystedt E, Kjolhede P, Gustavsson J, Nystrom FH \& Strålfors P 2005 Insulin resistance in human adipocytes downstream of IRS1 after surgical cell isolation, but at the level of phosphorylation of IRS1 in type 2 diabetes. FEBS Journal 272 141-151. (doi:10.1111/j.14321033.2004.04396.x)

DeMorrow S \& Francis H 2015 The novel growth factor, progranulin, regulates autophagy in a mouse model of cholestasis. FASEB Journal 29 (Supplement 1) 53.9. (available at (http://www.fasebj.org/content/29/ 1_Supplement/53.9)

Etemadi N, Webb A, Bankovacki A, Silke J \& Nachbur U 2013 Progranulin does not inhibit TNF and lymphotoxin- $\alpha$ signalling through TNF receptor 1. Immunology and Cell Biology 91 661-664. (doi:10.1038/icb. 2013.53)

Faustman D \& Davis M 2010 TNF receptor 2 pathway: drug target for autoimmune diseases. Nature Reviews. Drug Discovery 9 482-493. (doi:10.1038/nrd3030)

He Z, Ismail A, Kriazhev L, Sadvakassova G \& Bateman A 2002 Progranulin (PC-cell-derived growth factor/acrogranin) regulates invasion and cell survival. Cancer Research 62 5590-5596.

Hu Y, Xiao H, Shi T, Oppenheim JJ \& Chen X 2014 Progranulin promotes tumour necrosis factor-induced proliferation of suppressive mouse $\mathrm{CD}^{4(+)}$ Foxp $^{3(+)}$ regulatory T cells. Immunology 142 193-201. (doi:10. 1111/imm.12241)

Jian J, Zhao S, Tian Q, Gonzalez-Gugel E, Mundra JJ, Uddin SM, Liu B, Richbourgh B, Brunetti R \& Liu CJ 2013 Progranulin directly binds to the CRD2 and CRD3 of TNFR extracellular domains. FEBS Letter $\mathbf{5 8 7}$ 3428-3436. (doi:10.1016/j.febslet.2013.09.024)

Kawasaki N, Asada R, Saito A, Kanemoto S \& Imaizumi K 2012 Obesityinduced endoplasmic reticulum stress causes chronic inflammation in adipose tissue. Scientific Reports 2 799. (doi:10.1038/srep00799)

Kessenbrock K, Frohlich L, Sixt M, Lammermann T, Pfister H, Bateman A, Belaaouaj A, Ring J, Ollert M, Fassler R et al. 2008 Proteinase 3 and neutrophil elastase enhance inflammation in mice by inactivating antiinflammatory progranulin. Journal of Clinical Investigation $\mathbf{1 1 8}$ 2438-2447. (doi:10.1172/JCI34694)

Komatsu M, Waguri S, Ueno T, Iwata J, Murata S, Tanida I, Ezaki J, Mizushima N, Ohsumi Y, Uchiyama Y et al. 2005 Impairment of starvation-induced and constitutive autophagy in Atg7-deficient mice. Journal of Cell Biology 169 425-434. (doi:10.1083/jcb.200412022)

Kovsan J, Blüher M, Tarnovscki T, Klöting N, Kirshtein B, Madar L, Shai I, Golan R, Harman-Boehm I, Schön MR et al. 2011 Altered autophagy in human adipose tissues in obesity. Journal of Clinical Endocrinology and Metabolism 96 E268-E277. (doi:10.1210/jc.2010-1681)
Li M, Liu Y, Xia F, Wu Z, Deng L, Jiang R \& Guo FJ 2014 Progranulin is required for proper ER stress response and inhibits ER stress-mediated apoptosis through TNFR2. Cellular Signalling 26 1539-1548. (doi:10. 1016/j.cellsig.2014.03.026)

Liang H, Yin B, Zhang H, Zhang S, Zeng Q, Wang J, Jiang X, Yuan L, Wang CY \& Li Z 2008 Blockade of tumor necrosis factor (TNF) receptor type 1-mediated TNFA signaling protected wistar rats from diet-induced obesity and insulin resistance. Endocrinology 149 2943-2951. (doi:10. 1210/en.2007-0978)

Liu CJ \& Bosch X 2012 Progranulin: a growth factor, a novel TNFR ligand and a drug target. Pharmacology \& Therapeutics 133 124-132. (doi:10. 1016/j.pharmthera.2011.10.003)

Liu C, Li XX, Gao W, Liu W \& Liu DS 2014 Progranulin-derived Atsttrin directly binds to TNFRSF25 (DR3) and inhibits TNF-like ligand 1A (TL1A) activity. PLOS ONE 9 e92743. (doi:10.1371/journal.pone. 0092743)

Matsubara T, Mita A et al. 2012 PGRN is a key adipokine mediating high fat diet-induced insulin resistance and obesity through IL-6 in adipose tissue. Cell Metabolism 15 38-50. (doi:10.1016/j.cmet. 2011.12.002)

Mootha VK, Lindgren CM, Eriksson KF, Subramanian A, Sihag S, Lehar J, Puigserver P, Carlsson E, Ridderstråle M, Laurila E et al. 2003 PGC-1 $\alpha$ responsive genes involved in oxidative phosphorylation are coordinately downregulated in human diabetes. Nature Genetics 34 267-273. (doi:10.1038/ng1180)

Mortensen M, Ferguson DJ, Edelmann M, Kessler B, Morten KJ, Komatsu M \& Simon AK 2010 Loss of autophagy in erythroid cells leads to defective removal of mitochondria and severe anemia in vivo. PNAS 107 832-837. (doi:10.1073/pnas.0913170107)

Ost A, Svensson K, Ruishalme I, Brännmark C, Franck N, Krook H, Sandström P, Kjolhede P \& Strålfors P 2010 Attenuated mTOR signaling and enhanced autophagy in adipocytes from obese patients with type 2 diabetes. Molecular Medicine 16 235-246. (doi:10.2119/molmed. 2010.00023)

Patti ME, Butte AJ, Crunkhorn S, Cusi K, Berria R, Kashyap S, Miyazaki Y, Kohane I, Costello M, Saccone R et al. 2003 Coordinated reduction of genes of oxidative metabolism in humans with insulin resistance and diabetes: potential role of PGC1 and NRF1. PNAS 100 8466-8471. (doi:10.1073/pnas.1032913100)

Petersen KF, Befroy D, Dufour S, Dziura J, Ariyan C, Rothman DL, DiPietro L, Cline GW \& Shulman GI 2003 Mitochondrial dysfunction in the elderly: possible role in insulin resistance. Science $\mathbf{3 0 0}$ 1140-1142. (doi:10.1126/science.1082889)

Pospisilik JA, Knauf C, Joza N, Benit P, Orthofer M, Cani PD, Ebersberger I, Nakashima T, Sarao R, Neely G et al. 2007 Targeted deletion of AIF decreases mitochondrial oxidative phosphorylation and protects from obesity and diabetes. Cell 131 476-491. (doi:10.1016/j.cell. 2007.08.047)

Stralfors P \& Honnor RC 1989 Insulin-induced dephosphorylation of hormone-sensitive lipase: correlation with lipolysis and cAMP-dependent protein kinase activity. European Journal of Biochemistry 182 379-385. (doi:10.1111/j.1432-1033.1989.tb14842.x)

Tang W, Lu Y, Tian QY, Zhang Y, Guo FJ, Liu GY, Syed NM, Lai Y, Lin EA, Kong L et al. 2011 The growth factor progranulin binds to TNF receptors and is therapeutic against inflammatory arthritis in mice. Science $\mathbf{3 3 2}$ 478-484. (doi:10.1126/science.1199214)

Wils H, Kleinberger G, Pereson S, Janssens J, Capell A, Van Dam D, Cuijt I, Joris G, De Deyn PP, Haass C et al. 2012 Cellular ageing, increased mortality and FTLD-TDP-associated neuropathology in progranulin knockout mice. Journal of Pathology 228 67-76. (doi:10.1002/path.4043)

Wu S, Zhou B, Xu L \& Sun H 2009 IRS-2, but not IRS-1, can sustain proliferation and rescue UBF stabilization in InR or InR defective signaling of 32D myeloid cells. Cell Cycle 8 3218-3226. (doi:10.4161/cc.8.19.9759)

Yang L, Li P, Fu S, Calay ES \& Hotamisligil GS 2010 Defective hepatic autophagy in obesity promotes ER stress and causes insulin resistance. Cell Metabolism 11 467-478. (doi:10.1016/j.cmet.2010.04.005) 
Youn BS, Bang SI, Klöting N, Park JW, Lee N, Oh JE, Pi KB, Lee TH, Ruschke K, Fasshauer M et al. 2009 Serum progranulin concentrations may be associated with macrophage infiltration into omental adipose tissue. Diabetes 58 627-636. (doi:10.2337/db08-1147)

Zhou L, Zhang J, Fang Q, Liu M, Liu X, Jia W, Dong LQ \& Liu F 2009 Autophagy-mediated insulin receptor down-regulation contributes to endoplasmic reticulum stress-induced insulin resistance. Molecular Pharmacology 76 596-603. (doi:10.1124/ mol.109.057067)
Zhou B, Li H, Liu J, Xu L, Zang W, Wu S \& Sun H 2013a Intermittent injections of osteocalcin reverses autophagic dysfunction and endoplasmic reticulum stress resulting from diet-induced obesity in the vascular tissue via the NF-кB-p65-dependent mechanism. Cell Cycle 12 1901-1903. (doi:10.4161/cc.24929)

Zhou B, Li H, Xu L, Zang W, Wu S \& Sun H 2013b Osteocalcin reverses endoplasmic reticulum stress and improves impaired insulin sensitivity secondary to diet-induced obesity through nuclear factor-КB signaling pathway. Endocrinology 154 1055-1068. (doi:10.1210/en.2012-2144)

Received in final form 12 September 2015

Accepted 14 September 2015

Accepted Preprint published online 15 September 2015
Published by Bioscientifica Ltd. 